\title{
Concurrent near infrared imaging and spectroscopy
}

\author{
by D.A. Hutchins*, A. Saleem*, R.J. Green*, L.A.J. Davis*, C. Canal* and R. Gupta* \\ *School of Engineering, University of Warwick, Coventry CV4 7AL, UK, D.A.Hutchins@warwick.ac.uk
}

\begin{abstract}
This paper describes the use of near infrared techniques for both spectroscopy and imaging. The approach uses both techniques on the same sample, so that spectral information can be used in conjunction with an image to identify materials and objects. It is shown that the technique has applications to security screening, food quality inspection and biomedical measurements, using the ability of near infrared to penetrate through barriers.
\end{abstract}

\section{Introduction}

Near infrared signals have been used for many types of measurement, ranging from biomedical imaging to spectroscopy for chemical identification [1,2]. However, relatively little work has been published on the use of both imaging and spectroscopy to identify the presence of a particular material or object, perhaps hidden or buried within a second object. Examples would include the presence of an unwanted contaminant in a food, the identification of a subsurface lesion below the human skin, or the detection of an explosive or weapon hidden underneath a clothing layer. In all cases, the aim of the measurement is to find the region of interest, image it, and give some additional information concerning its properties. One way to do this is to exploit the properties of certain wavelengths of near infrared (NIR) signals for excellent penetration through non-conducting materials. In this paper, some experiments are described, which show that both imaging and spectroscopy can be performed, with applications to many interesting problems.

The work presented by the authors in a previous publication [3] established the feasibility of using NIR spectroscopy as a technique to detect specific chemicals across single intervening layers of clothing fabrics, for applications in possible security screening systems. In addition, uses have been identified in food quality screening [4]. The eventual deployment of this technique in a practical measurement application would depend on establishing performance benchmarks in situations that resemble a real testing environment, coupled with the ability to efficiently process and present the detection results to the operator in a user-friendly format. If the technique were to be utilized in a portable imaging system, it would need to be capable of operating in the presence of high levels of ambient noise affecting the relevant frequency band (for instance in the presence of direct sunlight). Provision would need to be made to enhance the signal-to-noise ratios (SNRs) adequately within the collected spectral data, so these could be used to drive the relevant calibration models that would be trained to detect the chemicals or features of interest within a given sample. The work presented here attempts to show that progress has been made in this area.

\section{Apparatus and experiment}

To help deal with this problem, the use of lock-in amplification has been investigated as a technique to provide such enhancement in SNR [5]. This approach was selected to enhance the detection and identification of spectra in a set of discrete measurements on individual samples. Experiments were performed both with and without the lock-in system being activated, and NIR spectra recorded, for samples of chemicals and food materials. Additionally, a scanning system was designed, using a two-dimensional software-controlled scan-stage, to perform cross-sectional NIR imaging of objects in through-transmission. The results were then displayed by means of contoured intensity images, based on data processed from the received spectra at each pixel location.

This approach was then extended further by using the spectral signature as input to neural network-based multivariate calibration models. This was used so that a particular chemical or material could be identified from its spectral signature. The initial experiments were based on collecting NIR spectra of glass cells containing chemicals of interest, hidden behind clothing fabric layers as a $2 \mathrm{D}$ scan, using a software-based lock-in amplifier to process the recorded spectra. The resulting spectra were then used offline in Matlab ${ }^{\mathrm{TM}}$ for training and testing neural networks designed for pattern-recognition/classification, in order to detect the presence of a particular chemical.

The equipment layout used for these experiments is shown schematically in Figure 1. The broad-spectrum halogen source could be modulated externally, and the modulation details used to control data collection from the Ocean Optics NIR256-2.1 spectrometer (860-2,000 nm). The sample or sample cell was held horizontally, and the modulated NIR broad spectrum signal transmitted through the object. The sample could then be moved horizontally using a PCcontrolled $X-Y$ stage. This allowed the collection of data from across an object, and the subsequent formation of an image, after processing of received spectral signals from different locations across the object. 


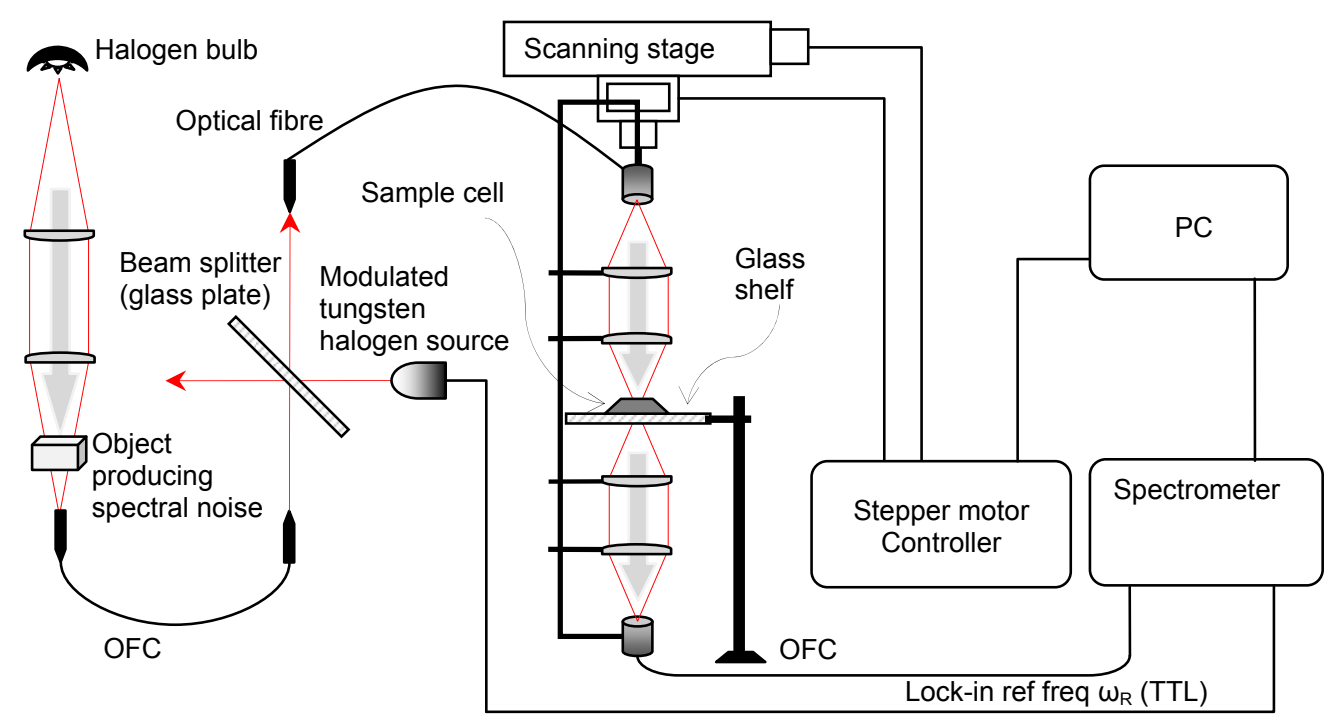

Fig. 1 - Schematic diagram of experimental arrangement used for collection and lock-in amplification of NIR spectral data.

In the case of liquids, the target chemical sample was held within a glass cell with a $1 \mathrm{~mm}$ wall thickness, and placed on top of a glass shelf attached to a 2D scan stage. The incident NIR signal was generated by means of a tungsten halogen light source, and was modulated at the lock-in amplifier reference frequency $\omega_{R}$. This frequency was software-generated on the PC as a $1-5 \mathrm{~Hz}$ square wave, and routed to the source via the spectrometer as a TTL signal switching the source on/off at the frequency $\omega_{R}$. A second component could also be added to this signal as a source of spectral noise in order to test the ability of the lock-in process to recover the original signal from such noise. This component was generated by a broadband halogen source, and transmitted through a test object such as a clear PET bottle so as to acquire the spectral profile of that object. The two components were combined together using a beam splitter arrangement, and delivered to the scanning stage via a low-OH content optical fibre cable (OFC). The NIR beams were conditioned throughout the process by means of an arrangement of optical lenses comprising collimating and focusing lenses. The signal transmitted through the sample in the scan stage was routed via an OFC to the spectrometer, and the spectrometer output was processed with optional lock-in amplification on the PC. All absorption spectra were recorded with reference to the uninterrupted signal intensity (with no target present) from the tungsten halogen source, with the exception of scans performed in the presence of fabric layers, in which case intensity across the relevant fabric layers was taken as reference.

The chemicals used in this case are listed in table 1. Scans were carried out to image these chemicals both separately and simultaneously in sets of two. In the latter case, the two cells containing the chemicals were placed alongside each other on the glass shelf, and the scan dimensions adjusted to cover both the cells. Further scans were carried out with the chemical/s covered by a layer of white polyester fabric, to investigate the possibility of lock-in amplification and detection across such layers. Also shown in table 1 are the meat samples chosen for study. Meat was chosen as an excellent example of a material that could mimic the applications of food inspection and also biomedical imaging. The materials studied were lamb, chicken and prosciutto (a cured, dried ham).

Table 1: Chemicals used to investigate lock-in amplification and spectroscopic imaging.

\begin{tabular}{|l|l|l|l|}
\hline Chemical & Formula & Physical state & Concentration \\
\hline Ammonium nitrate & $\mathrm{NH}_{4} \mathrm{NO}_{2}$ & $\begin{array}{l}\text { Aqueous } \\
\text { solution }\end{array}$ & $1.5 \mathrm{~g} \cdot \mathrm{ml}^{-1}$ \\
\hline Ethanol & $\mathrm{C}_{2} \mathrm{H}_{5} \mathrm{OH}$ & Liquid & $100 \% \mathrm{v} / \mathrm{v}$ \\
\hline Hydrogen peroxide & $\mathrm{H}_{2} \mathrm{O}_{2}$ & $\begin{array}{l}\text { Aqueous } \\
\text { solution }\end{array}$ & $30 \% \mathrm{v} / \mathrm{v}$ \\
\hline Distilled water & $\mathrm{H}_{2} \mathrm{O}$ & Liquid & $99 \% \mathrm{v} / \mathrm{v}$ \\
\hline
\end{tabular}

As stated above, the optical apparatus held in the scanning stage (including transmitter, receiver and associated lenses in figure 1) could be scanned in the horizontal plane following a raster scan profile, to obtain spectral data at pre-defined intervals along the surface of sample. This was done so as to investigate the feasibility of the technique in a two-dimensional spectroscopic imaging application. The spectral data collected using the apparatus of Figure 1 could be treated in two ways, in that the spectral data could be collected and processed with or without the use 
of the lock-in (which was not essential for some measurements in meat samples). In addition, data could be input into a neural network-based multivariate calibration model [6], trained for pattern recognition/classification, to identify the presence of certain chemicals of interest. If the lock-in was not used, then the full 200 separate wavelength channels from the spectrometer could be used. With the lock-in enabled, the wavelength range included in the measurements spanned 40 channels from $850 \mathrm{~nm}$ to $2,200 \mathrm{~nm}$.

\section{Results}

\subsection{The use of the lock-in technique}

In an initial set of measurements, a cell containing distilled water was used as the target, while the signal transmitted through a PET bottle (via the beam-splitter arrangement shown in figure 1) represented a source of unwanted additive spectral noise. Figure 2(a) shows the spectrum of water recorded with the interfering signal from the PET bottle switched off. This profile was modified as seen in figure 2(b) with the introduction of the PET signal, which gave rise to a characteristic peak at $1,675 \mathrm{~nm}$ [7]. As expected, the influence of the PET spectrum was effectively eliminated with the activation of the lock-in amplifier, as the signal carrying the noise spectrum was not modulated with lock-in reference frequency. As seen in figure 2(c), the resulting spectrum closely resembled the original, thus proving the effectiveness of lock-in amplification in cancelling out any spurious spectral content introduced by unwanted sources.

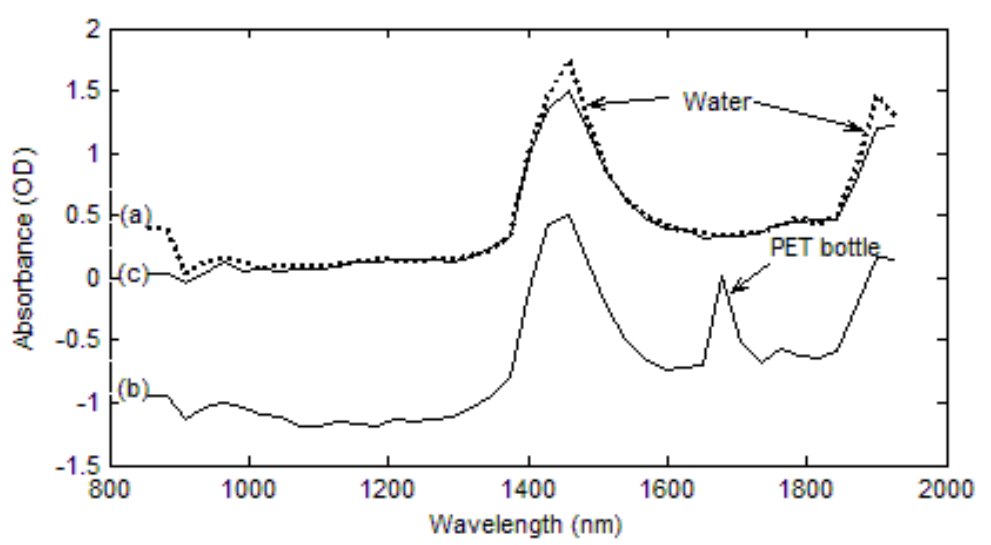

Fig. 2: NIR Spectra collected with point-scans across a cell containing distilled water. (a) Water without additive spectral noise; (b) Spectral noise from a PET bottle introduced in (a); (c) Result of lock-in amplification of spectra in (b).

\subsection{Reduction of significant noise and use of multivariate calibration models}

The first experiments simulated the case where the spectral data were completely buried in noise, which was deemed to be a highly probable scenario in practical screening applications where measurements are often performed in environments with high ambient noise. It was expected that the detection might fail in such a case in the absence of lockin amplification.

The simulation was carried out by adding high-amplitude software-generated uniform white noise to all spectrometer wavelength channels. Lock-in amplification of the resulting spectra was performed in the presence of increasing noise amplitudes, and the criterion for successful extraction of the signal from noise was based on the result of neural network-based classification. It was seen that the classification algorithm remained completely resilient to reduction in SNR of up to $-40 \mathrm{~dB}(\mathrm{SNR}=1: 100)$. Figure 3 shows the results of a recorded set of spectra of distilled water. With no noise added, figure 3(a), the usual NIR spectrum of water was recorded. With the SNR set at $-60 \mathrm{~dB}$, figure 3(b), the distinctive spectral features of water were lost in the noisy spectra. With the activation of the lock-in amplifier, however, spectral distortion was significantly diminished, and the spectral profile was recovered to a state close to the original form, as seen in figure 3(c). This shows the usefulness of a lock-in approach for noisy environments.

Scans were now performed to illustrate the usefulness of the combined use of the lock-in system, together with a chemical classification process using multivariate calibration models. For this test, the scanning system was used to scan over the sample, in this case in the form of a cell containing distilled water. The aim was to form an image of the area containing water only, based on analysing the signals to estimate the concentration of water present, based on its spectrum. Figure 4 shows intensity images depicting the outcome of the classification process for three scenarios: (a) the spectrum with no noise added; (b) a spectrum with additive noise to give a resultant SNR of -60dB, and (c) the result when the lock-in was used. In each case, the chemical was identified via the neural network-based multivariate calibration models. It can be seen that in the absence of lock-in amplification, the introduction of noise at a SNR of -60dB completely nullified the detection process (figure 4(b)), while lock-in amplification allowed recovery of the spectral data from the noise, and the detection to proceed with results closely matching those obtained in the absence of noise (figures 4 (a) and 4(c)). 


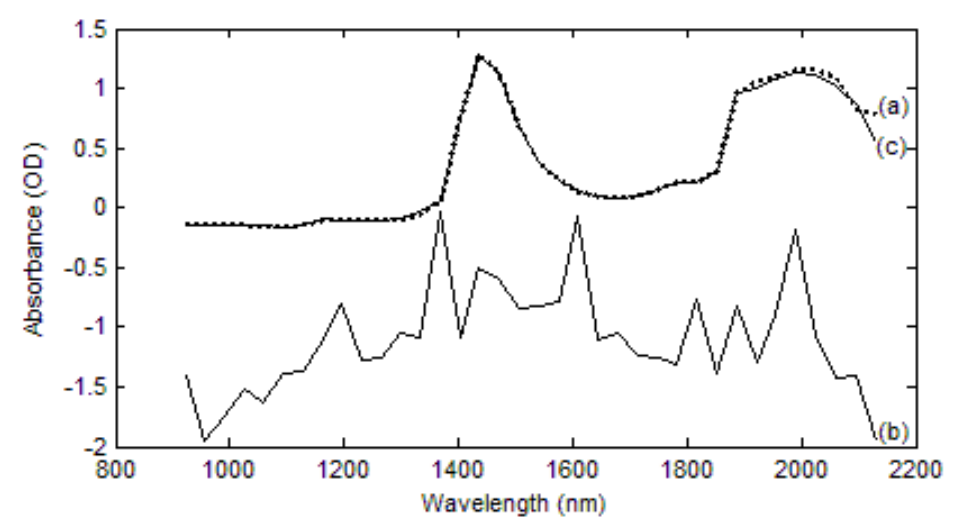

Fig. 3: NIR spectra recorded at a single location for a cell containing distilled water. (a) Water without additive spectral noise; (b) Spectrum with white noise deliberately added (SNR = -60 dB); (c) Result of lock-in amplification of spectrum in (b), where the original spectrum has been recovered.

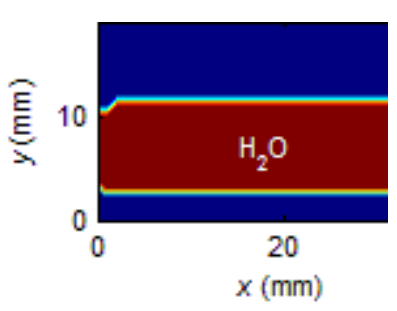

(a)

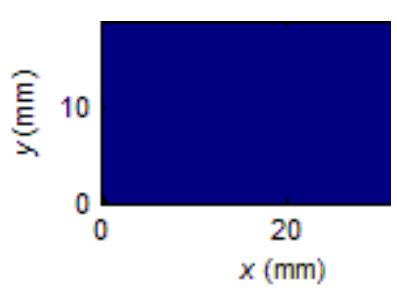

(b)

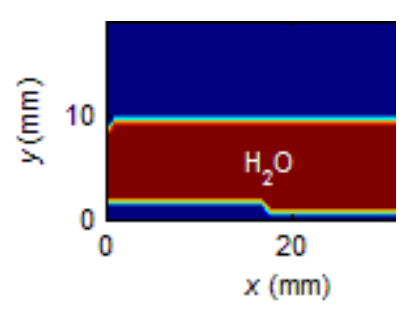

(c)

Fig. 4: Neural network-based spectral pattern classification of 2D scans across a cell containing distilled water. Classification results with (a) original spectra (no additive noise), (b) spectra collected in the presence of uniform white noise $(S N R=-60 d B)$, and (c) noisy spectra with lock-in amplification.

\subsection{Scans with combinations of different chemicals}

The technique was used to scan and image target sets comprising two cells in each case, where each cell contained a different chemical. The test spectra collected were buried in software-generated spectral noise so as to maintain an SNR of $-60 \mathrm{~dB}$. The same processing methodology as above was employed, including lock-in amplification followed by neural network-based classification, to identify the chemicals in the cells. The three chemicals used in the scans included solutions of ethanol, hydrogen peroxide and ammonium nitrate in the concentrations given earlier in Table 1. In the first instance, cells containing hydrogen peroxide and ethanol were scanned simultaneously. Intensity images depicting classification results obtained with the spectra collected in each of the three scanned scenarios are shown in figure 5 .

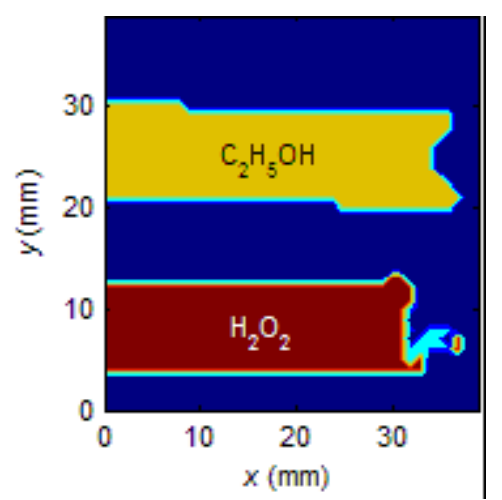

(a)

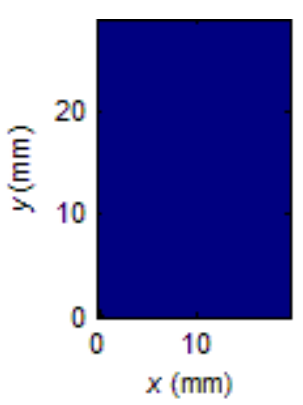

(b)

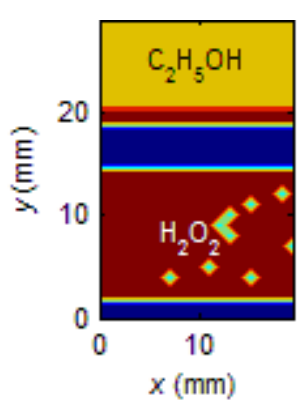

(c)

Fig. 5: Neural network-based spectral pattern classification of 2D scans across two cells containing hydrogen peroxide and ethanol. Classification results with (a) original spectra (no additive noise), (b) spectra collected in the presence of uniform white noise $(S N R=-60 \mathrm{~dB})$, and (c) noisy spectra with lock-in amplification.

As seen, the scan dimensions were reduced after the first scan (figure 5(a)), while still covering parts of both the cells. This was prompted by the need to reduce the time taken to complete each scan. The time consumed in the first scan, with a grid size of $40 \mathrm{~mm} \times 40 \mathrm{~mm}$, step size of $1 \mathrm{~mm}$, and scan rate of $2 \mathrm{sec} /$ point, was approximately one hour, 
which was reduced to 20 mins with the reduced grid size of $20 \mathrm{~mm} \times 30 \mathrm{~mm}$. (Note that in a practical system for personal screening, a rapid scanning set-up could be used, for instance with an optically-scanned light source or large area illumination). It is seen that a small number of spectra in figures 5(a) and (c) were misclassified as ammonium nitrate (coloured cyan), while the spectra along the edge of the ethanol cell in figure 5(c) were misclassified as hydrogen peroxide. However, the proportion misclassified was deemed to be within acceptable margin of error, as more than $90 \%$ of the spectra were correctly classified in both these cases. Finally, none of the spectra buried in noise were correctly classified in the absence of lock-in amplification (figure 5(b)), which was consistent with the previous findings.

A scan was also performed with ammonium nitrate replacing ethanol alongside hydrogen peroxide. The corresponding intensity images depicting classification results are shown in figure 6 . It can be seen that, as in the previous case, a small number of spectra were misclassified. The most noticeable of these were along the border of the ammonium nitrate cell in figure 6(c), which were misclassified as ethanol (coloured yellow). However, the overwhelming majority of spectra were correctly classified, except those collected in the presence of high amplitude noise without lockin amplification (figure 6(b)), as expected.

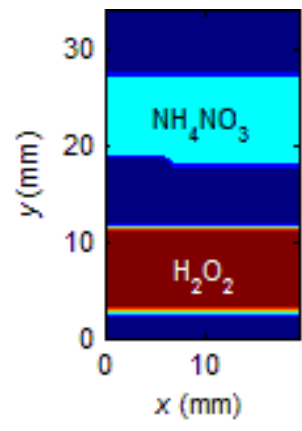

(a)

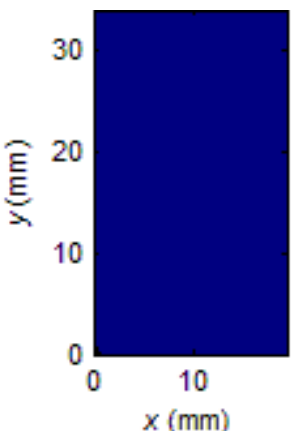

(b)

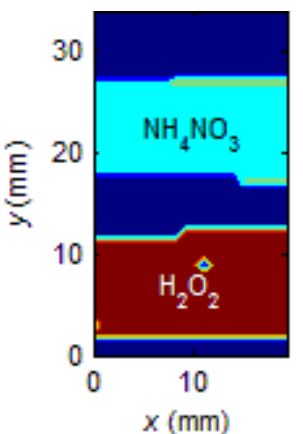

(c)

Fig. 6: Neural network-based spectral pattern classification of 2D scans across two cells containing hydrogen peroxide and ammonium nitrate. Classification results with (a) original spectra (no additive noise), (b) spectra collected in the presence of uniform white noise $(S N R=-60 \mathrm{~dB})$, and (c) noisy spectra with lock-in amplification.

Although not shown here, other experiments have been performed which demonstrate the detection of such chemicals under clothing layers. The use of the classification algorithms was found to be very important, and it has been shown that the same chemicals described above can be identified when covered by a fabric layer. This is important in applications such as personal security screening.

\subsection{Experiments on meat samples}

Based on the above results, experiments have also been performed to investigate the performance of NIR spectroscopy for food inspection and biomedical applications. An important aspect of this is to identify the parameters that govern propagation through muscle tissue and fat layers. The apparatus of figure 1 has thus been used to record spectra and produce images for these biological samples.

Figure 7(a) shows the spectrum recorded far a lean (i.e. non-fatty) sample of lamb. It can be seen immediately that that the spectrum is dominated by that of water, with the characteristic NIR absorption peaks seen in the results above. It can thus be concluded that the presence of water is the variable that will affect spectral recording in lamb muscle. This can be compared to the results obtained through a sample of fat from the same meat sample, the results of which are shown in figure $7(\mathrm{~b})$. It can be seen now that the fat layer has no observable major features, but has an increased absorption coefficient with increasing wavelength. Note, however, that the absorption coefficient range recorded for the fat sample is lower than that in muscle tissue in general, but higher at certain wavelengths. This interesting information could possibly be used to discriminate lean tissue from fat.

- With such a clear distinction in the spectra, it was found in these samples that no lock-in or multivariate calibration was required. The samples were sufficiently transparent to NIR signals in the $900-2,200 \mathrm{~nm}$ range that neither was required for the detection of recognisable spectra, and the formation of images from them. Another example is that of prosciutto, a meat that is thinly-sliced dried and cured pork. This was tested in the same way, and the spectra of lean meat and fat recorded. The results are shown in figure 8 , where it will be evident that the lean meat still contained the spectrum of water as the dominant feature. As also seen for lamb, the absorption coefficient values were in general at a lower value. To illustrate this, an image was recorded, with the apparatus of figure 1 used to scan the NIR beam across a sample of prosciutto, which contained an outer area of lean meat which surrounded an area of fat, with a region in-between of mixed fat and lean meat. The image that resulted is shown in figure 8(c), where the amplitude scale corresponds to the relative absorption 
coefficients seen on the spectra at the wavelengths $1921 \mathrm{~nm}$ and $1436 \mathrm{~nm}$. Here, lower absorption (and hence greater NIR transmission) is represented by a darker grey-scale shade. The area of fat is identified easily.

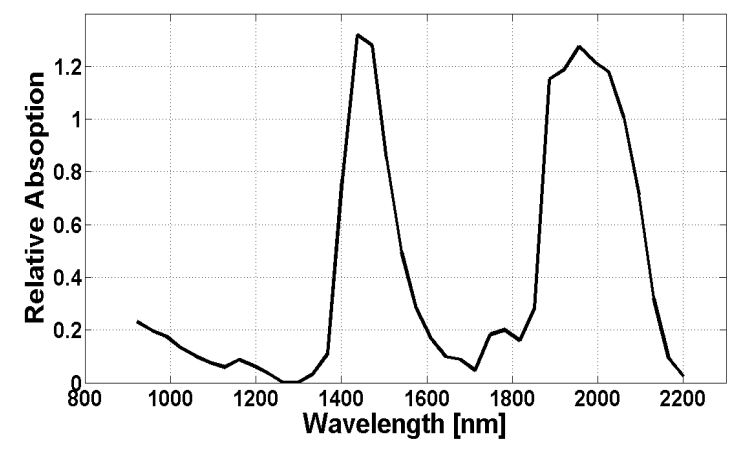

(a)

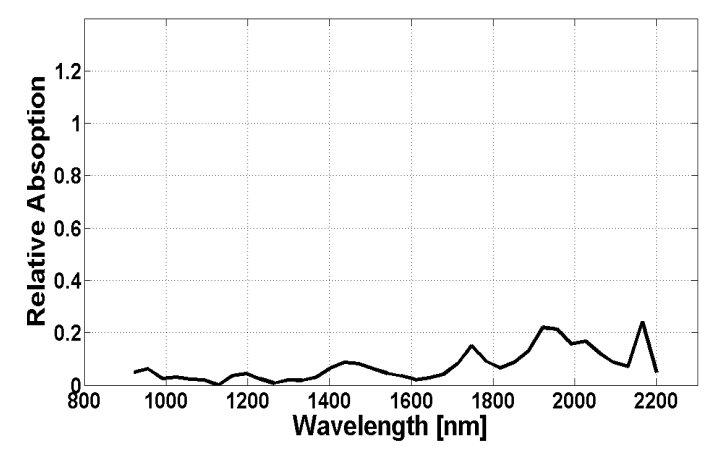

(b)

Fig. 7: Absorption spectra of (a) lean lamb meat and (b) lamb fat.

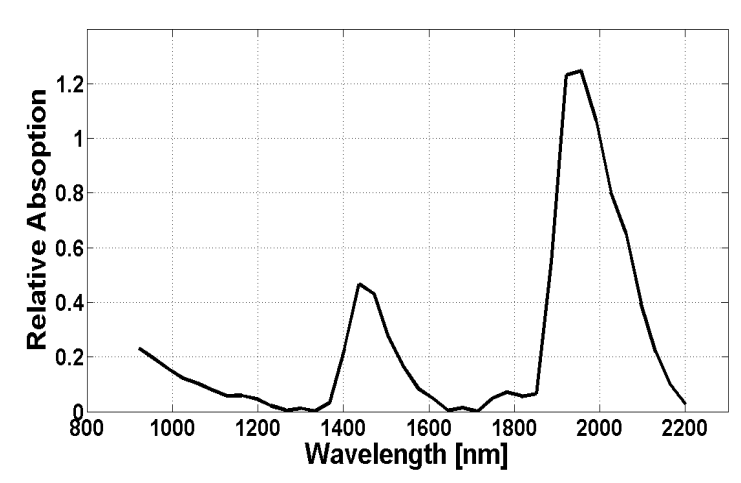

(a)

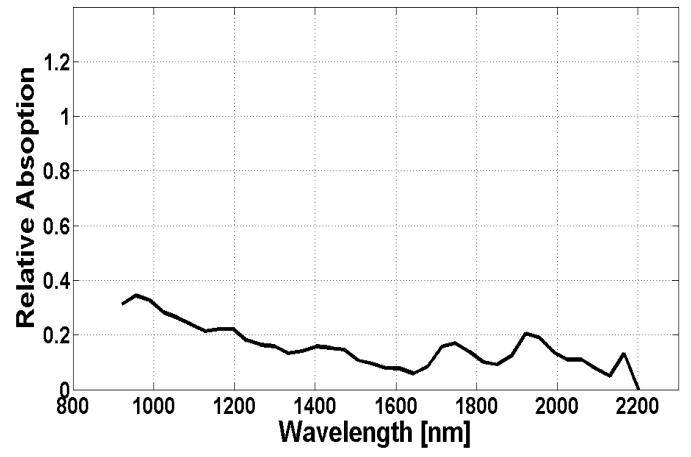

(b)

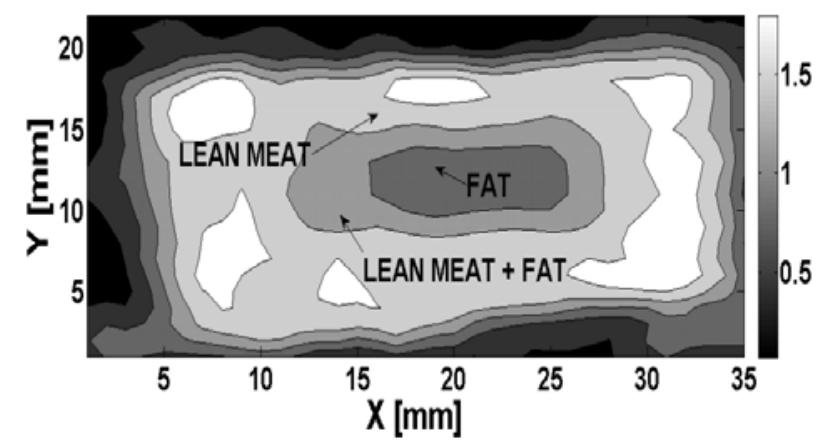

(c)

Fig. 8: Absorption spectra of (a) lean prosciutto and (b) prosciutto fat. (c) An NIR through-transmission image of a prosciutto sample, having lean meat surrounding a region of fat.

\section{Conclusions}

The work has demonstrated that NIR spectra can be recorded in through-transmission through a range of different materials. In materials with a high absorption, it was found necessary to use several techniques in order to be able to record NIR spectra, and also to use these to identify a particular chemical species. It was found that the use of a lock-in amplifier system, implemented in software, was able to control the data collection from the spectrometer in a way 
that provided significant improvements in SNR. With the further addition of a neural networks-based chemical identification algorithm, certain chemicals could be identified, even when the SNR had reached a level of $-60 \mathrm{~dB}$.

In the case of animal products, it is found that the predominant spectrum in muscle tissue is that of water, which has two major absorption peaks within the 1,400 nm - 2,200 nm wavelength range. These dominate absorption within the lean meat samples investigated. Conversely, absorption within fat is at a lower level, without any major absorption peaks within the same wavelength range.

It is hoped in the future to use the various techniques shown here for sensitive biomedical imaging. In particular, the combination of spectral imaging, lock-in noise reduction and multivariate calibration seems to be a promising way forward.

\section{REFERENCES}

[1] Siesler H. W., Ozaki Y., Kawata S. and Heise H. M., "Near-infrared spectroscopy: principles, instruments, applications". Wiley-VCH, 2006.

[2] Liao S. M., Gregg N. M., White B. R., Zeff B. W., Bjerkaas K. A., Inder T. E. and Culver J.P., "Neonatal hemodynamic response to visual cortex activity: high-density near-infrared spectroscopy study". Journal of Biomedical Optics, vol. 15, p. 026010 (9 pp.), 2010.

[3] Saleem A., Canal C., Hutchins D.A. and Green R.J., "Techniques for quantifying chemicals concealed behind clothing using near infrared spectroscopy", Anal. Methods vol. 3, 2298 - 2306, 2011.

[4] Pallav P., Diamond G.G, Hutchins D.A., Green R.J. and Gan T.H., "A near infrared (NIR) technique for imaging food materials". J. Food Sci., vol. 74, pp. E23-E33, 2009.

[5] M. J. Holcomb and W. A. Little, "Cascading lock-in amplification: application to wavelength modulation spectroscopy". Review of Scientific Instruments, vol. 63, pp. 5570-5, 1992.

[6] Sharma S., "Applied multivariate techniques". J. Wiley, New York, 1996.

[7] Rodgers J. and Beck K., "NIR Characterization and Measurement of the Cotton Content of Dyed Blend Fabrics," Textile Research Journal, vol. 79, pp. 675-686, 2009. 